\title{
Les effets du rayonnement des étoiles sur la matière interstellaire
}

\author{
P. Brechignac
}

\author{
Laboratoire de Photophysique Moléculaire, CNRS, Université Paris-Sud, bâtiment 210, \\ 91405 Orsay cedex, France
}

\begin{abstract}
Résumé : Le rayonnement des étoiles joue un rôle fondamental dans l'évolution physico-chimique de la matière interstellaire qui permet à de nouvelles étoiles et à de nouveaux systèmes planétaires de se former. Le rayonnement UV ou VUV agit sur la composante gazeuse du milieu principalement par photoionisation, pour certains atomes et les grosses molécules notamment, et par photodissociation sur la plupart des molécules, en particulier de petite taille. Il agit sur la composante solide et réfractaire en chauffant le milieu soit par absorption directe, soit en y injectant des électrons "chauds" créés par effet photoélectrique. Dans les régions denses et froides il provoque une photolyse des manteaux de glaces moléculaires condensées à la surface des grains. Dans les coeurs denses des nuages où l'on trouve de jeunes étoiles en formation, le rayonnement $X$ présent est susceptible de modifier profondément la nature chimique du matériau constituant les grains interstellaires.
\end{abstract}

\section{INTRODUCTION AU MILIEU INTERSTELLAIRE}

C'est au sein d'un milieu extrêmement ténu, froid, et de très vaste étendue que se forment les étoiles, au terme d'une évolution dynamique et chimique très complexe. C'est également dans ce milieu que se retrouve la matière éjectée par les étoiles en fin d'évolution. L'interaction entre le rayonnement des étoiles et la matière interstellaire qui les environne est un des moteurs essentiels de l'évolution chimique de celleci. Cette évolution est essentielle car elle détient la clé du refroidissement du milieu interstellaire (MIS), lequel conditionne la phase d'effondrement gravitationnel.

La plus grande partie de la masse du MIS se trouve concentrée dans des nuages de gaz et de poussière. La composante gazeuse représente à elle seule $99 \%$ de la masse totale. La phase solide, constituée par des petites particules, les grains interstellaires, joue néanmoins un rôle capital dans l'évolution chimique du MIS car elle offre un support pour des processus catalytiques et/ou des réactions en phase hétérogène. La troisième composante du MIS est un champ de rayonnement intense et dont l'énergie des photons est assez élevée pour constituer un environnement hostile [1].

La présence d'une distribution de taille des grains de poussière est responsable de l'essentiel de "l'extinction" interstellaire, c'est-à-dire l'atténuation de la lumière en provenance des étoiles, qui est - en première approximation - d'autant plus efficace que la longueur d'onde du rayonnement diminue. C'est ainsi que l'on pourra observer des régions d'autant plus denses que l'on utilise un rayonnement de plus grande longueur d'onde. C'est une des raisons qui ont motivé le lancement du satellite d'observation infrarouge ISO dont la période d'activité s'est étendue de novembre 1995 à mai 1998.

Le recyclage de la matière interstellaire dans les étoiles est responsable de l'observation simultanée de divers types d'objets astrophysiques pouvant se situer à des stades d'évolution très différents. Il en résulte des différences considérables dans la nature des conditions physiques suivant les régions observées : densités, températures, densité de photons et répartition spectrale.

Dans le milieu "diffus" (loin des condensations) où les densités restent comprises entre 1 et 100 particules $/ \mathrm{cm}^{3}$, le premier effet du rayonnement est de photoioniser l'atome H. L'absorption cumulée est tellement importante que le champ de rayonnement interstellaire moyen ne comporte plus de photons d'énergie supérieure à $13.6 \mathrm{eV}$. La conséquence est que le milieu est autoprotégé contre les photons de grande énergie. Par contre dans les régions où l'activité de formation d'étoiles est grande, en particulier dans les "coeurs denses", le spectre du rayonnement peut s'étendre dans le domaine X. Dans les nuages 
moléculaires, où les densités peuvent atteindre $10^{6}$ particules $/ \mathrm{cm}^{3}$, les molécules les moins volatiles se condensent à la surface des grains en formant ainsi un " manteau " de glace.

\section{LE DOMAINE UV - VUV}

On présente ci-dessous quelques exemples parmi les plus importants pour illustrer les effets de l'irradiation sur les deux composantes de la matière interstellaire : le gaz et les grains. On notera que l'effet du rayonnement UV ou VUV sera souvent plus facile à détecter dans le domaine infrarouge (ISO notamment).

\subsection{Le gaz}

Dans le domaine UV, les phénomènes principaux sont la photoionisation des atomes ou des molécules, la photodissociation des petites molécules, la photofragmentation des grosses molécules (en particulier pour les hydrocarbures aromatiques polycycliques).

Le refroidissement du milieu se fait principalement par échappement de photons qui empruntent leur énergie à l'énergie cinétique "thermique" du gaz. Parmi les agents de ce refroidissement les transitions de structure fine des atomes, les transitions rotationnelles de petites molécules comme $\mathrm{H}_{2}$ ou $\mathrm{CO}$, jouent un rôle important. Parmi les trois atomes "lourds" (hormis $\mathrm{H}$ et $\mathrm{He}$ ) les plus abondants que sont le carbone, l'azote et l'oxygène, seul le carbone a un potentiel d'ionisation nettement inférieur à 13.6 $\mathrm{eV}$ : le premier effet du champ de rayonnement UV sera donc de photoioniser $\mathrm{C}$ pour former $\mathrm{C}^{+}$, alors que $\mathrm{O}$ et $\mathrm{N}$ ne le seront pas. C'est la raison pour laquelle le satellite ISO a récemment observé en tant qu'agents de refroidissement la transition de structure fine de $\mathrm{C}^{+}$à 158 microns dans les régions de faibles densités que constituent les "cirrus", et la transition de $\mathrm{O}$ (neutre) à 63 microns dans la région dense du nuage moléculaire SaggitariusB2 (entre autres). Parmi les espèces moléculaires pouvant être photoionisées directement la plupart sont polyatomiques. On trouve en particulier la famille des hydrocarbures aromatiques polycycliques ( $\mathrm{PAH}=$ polycyclic aromatic hydrocarbons), dont le potentiel d'ionisation décroît avec la taille. Dans ce cas, néanmoins, il faut prendre en compte la compétition avec la photodissociation.

La molécule d'hydrogène $\mathrm{H}_{2}$ est de très loin la plus abondante dans les nuages interstellaires. Les effets du rayonnement sur cette molécule présentent donc un intérêt essentiel. De fait, elle présente un cas très particulier de photodissociation radiative spontanée. En effet les états électroniques $B$ et $C$, pompés optiquement par le champ de rayonnement interstellaire, ne sont pas prédissociés et ne peuvent se désexciter que radiativement. La plupart de ces cycles d'absorption - émission contribuent à redistribuer la population sur les états rovibrationnels de l'état électronique fondamental $\mathrm{X}$, mais une fraction de l'ordre de $10 \%$ conduit à la dissociation par suite de l'émission lié - continuum détectée entre 1150 et $1700 \AA$ [2] La population rovibrationnelle formée dans les régions "dominées par les photons" (PDR = photon dominated regions) est elle-même observée dans l'infrarouge (ISO), sur les transitions de rotation pure [3], ou de vibration-rotation [4].

Les enveloppes circumstellaires, régions qui entourent les atmosphères des étoiles qui rejettent, en fin de vie, une grande quantité de matière dans le milieu environnant, sont considérées comme des lieux d'intense production de grains. Si le matériau de l'étoile est plus riche en oxygène qu'en carbone, la formation de $\mathrm{CO}$ laissera un excès d'oxygène (étoiles oxygénées) quii se retrouvera dans des grains de type "silicates". Par contre si c'est l'inverse (étoiles carbonées) le carbone se retrouvera dans l'acétylène $\mathrm{C}_{2} \mathrm{H}_{2}$, susceptible d'être photodissociée, puis dans les PAHs et diverses formes de grains carbonés [5]. La photodissociation de $\mathrm{C}_{2} \mathrm{H}_{2}$ fait l'objet d'études en laboratoire afin d'évaluer les taux de destruction dans le MIS, ainsi que les rapports de branchement vers les différents fragments $\mathrm{C}_{2} \mathrm{H}, \mathrm{C}_{2}, \mathrm{CH}$. Les états de Rydberg de cette molécule sont les principaux responsables de la photodissociation, cependant l'efficacité du processus dépend de manière cruciale des caractéristiques propres à chacun de ces états [6]. 
Le principal effet du rayonnement UV-VUV sur les PAHs est de nous permettre de les observer en infrarouge grâce à un processus de conversion interne intramoléculaire. Au cours de ce processus l'énergie d'un photon unique absorbé sur l'une des transitions électroniques est convertie en énergie vibrationnelle, qui est ensuite évacuée par la molécule (qui se refroidit) sous forme de rayonnement infrarouge [7] . L'apport des observations récentes faites à partir d'ISO a considérablement enrichi notre connaissance actuelle de cette composante interstellaire qui contient environ $15 \%$ du carbone cosmique disponible. Elles ont notamment confirmé la stabilité du spectre infrarouge pour des conditions où l'intensité du champ de rayonnement varie sur plus de trois ordres de grandeur [8]. Ceci a été possible grâce à de nombreuses études de laboratoire menées sur ces espèces durant les dix dernières années. La photofragmentation des PAHs fait elle aussi partie de ces études en laboratoire. Il s'agit d'un processus unimoléculaire, faisant suite à une redistribution statistique de l'énergie injectée dans la molécule sur tous les modes vibrationnels accessibles. On observe notamment l'éjection de fragments polyatomiques, comme $\mathrm{C}_{2} \mathrm{H}_{2}$ par exemple [9].

\subsection{Les grains}

Le principal effet du rayonnement des étoiles sur la composante solide du MIS est de chauffer le milieu. Il faut néanmoins distinguer le chauffage des grains eux-mêmes, résultant des propriétés d'absorptivité émissivité et de diffusion des matériaux constitutifs, et le chauffage (indirect) de la phase gazeuse environnante, induit par l'éjection d'électrons chauds par effet photoélectrique à la surface des grains. Il va de soi que l'évaluation correcte de cet effet passe par une bonne connaissance de la nature des diffétents types de grains : silicates, carbone amorphe, charbons, matériau graphitique, manteaux de glaces moléculaires,... La photoionisation des PAHs de grande taille participe d'ailleurs à ce processus [10].

Dans les régions plus denses où la surface des grains interstellaires est recouverte soit d'une fine couche moléculaire adsorbée, soit d'un manteau de glaces moléculaires condensées, d'autres processus peuvent intervenir. La photodésorption de molécules neutres ou d'ions est un processus qui peut modifier l'équilibre gaz - grains par rapport aux données purement thermodynamiques. Les données de laboratoire sur ce phénomène sont encore trop peu nombreuses. Par contre des simulations de laboratoire ont été réalisées pour identifier les produits de réaction formés dans la photolyse des manteaux de glaces dont certains sont inattendus comme les ions $\mathrm{OCN}^{-}$et $\mathrm{NH}^{+}{ }^{+}$[11]. Cette photochimie hétérogène apporte des solutions à la formation de certaines molécules pour lesquelles on ne trouve pas de schéma de formation plausible en phase gazeuse, telles que $\mathrm{CO}_{2}$.

Enfin il est possible que l'irradiation prolongée en UV-VUV des grains silicatés soit à l'origine de la transformation allotropique récemment mise en évidence : la spectroscopie infrarouge réalisée avec ISO révèle une transformation progressive des silicates amorphes en silicates cristallins, de type forstérite par exemple, en fonction de l'âge des objets observés [12].

\section{LE DOMAINE $X$}

Le domaine des rayons $\mathrm{X}$ n'est pas aussi universellement présent dans le MIS que ne l'est le rayonnement UV-VUV. Il est associé aux régions denses où l'on peut détecter, par cartographie et mesure de température de couleur en infrarouge, des étoiles très jeunes, ou des protoétoiles en formation . Le nuage principal autour de l'étoile $\rho$-Ophiuchi offre un bon exemple de cette circonstance [13]. Dans cette phase primitive de l'évolution stellaire, l'étoile accrète de la matière en provenance d'un disque circumstellaire, et les sursauts d'émission $\mathrm{X}$ pourraient être produits par la "connexion magnétique" entre l'étoile et le disque [14]. Le domaine d'énergie de photons mise en jeu est de l'ordre du keV. La principale prédiction des modèles pour de telles régions est que le taux d'ionisation peut demeurer élevé même dans des régions de forte densité, contrairement à ce qui se passe dans des régions dépourvues de rayonnement $\mathrm{X}$ [15]. 
Les effets sur la matière interstellaire du rayonnement dans ce domaine sont plus difficiles à appréhender. Des expériences d'irradiation d'échantillons de divers types de matériaux pouvant servir d'analogues de grains interstellaires commencent à être réalisées. Il semble que les silicates cristallins et le graphite ne subissent pas de transformation; les charbons subissent une déshydrogénation; les PAHs de petite taille subissent une rupture partielle des cycles aromatiques, tandis que les plus gros ( 24 atomes $\mathrm{C}$ ) ne montrent qu'une déshydrogénation [16]. Des études de ce type devraient être poursuivies dans des conditions contrôlées dans la mesure où elles sont susceptibles d'apporter un éclairage neuf sur de grandes questions comme l'évolution du caractère cristallin/amorphe de la matière silicatée, ou la graphitisation de la matière carbonée.

\section{CONCLUSION}

Les effets du rayonnement des étoiles sur la matière interstellaire sont donc nombreux et variés. Ils dépendent de la composition spectrale du champ de rayonnement local (UV-VUV / X) et de la nature physique de la matière présente (gaz, atomes, molécules/grains), et donc à double titre de la diversité des types d'objets que l'on rencontre dans le MIS. Leur étude repose sur un va-et-vient permanent entre résultats d'observations astronomiques à toutes longueurs d'onde et interprétation à l'aide de mesures en laboratoire ou de modélisations théoriques.

En France, le Programme National PCMI ${ }^{1}$ coordonne l'activité de physiciens et physico-chimistes de laboratoire, de théoriciens de chimie quantique et dynamique moléculaire, d'astronomes observateurs, et de concepteurs de modèles astrophysiques, pour faire progresser ce champ de recherche par nature interdisciplinaire, qui occupe un rôle central entre la physique des galaxies et celle de la formation de systèmes planétaires. Des avancées remarquables ont été réalisées qui placent la communauté française dans un très bon rang au niveau international.

\section{Références}

1. J.Lequeux and E.Roueff, Phys. Rep. 200, 241 (1991)

2. A.N.Witt, T.P.Stecher, T.A.Boroson, R.C.Bohlin, Ap. J. 336, L21 (1989)

3. D.A.Neufeld, G.J.Melnick, M.Harwit, Ap. J. 506, L75 (1998)

4. R..Timmermann, F.Bertoldi, C.M.Wright,. S.Drapatz, B.T.Draine, L.Haser, A.Stemberg, A\&A, 315, L281 (1996)

5. S.Lafont, R.Lucas, A.Omont, A\&A 106, 201(1982); I.Cherchneff, A.E.Glassgold, Ap.J. 419, L41 (1993)

6. N.Shafizadeh, J.H.Fillion, D.Gauyacq, S.Couris, Phil.Trans. Roy.Soc.A355, 1637 (1997)

7. Polycyclic Aromatic Hydrocarbons and Astrophysics, edited by A.Léger, L.d' Hendecourt and N.Boccara, Reidel (1987).

8. F.Boulanger et al, ASP Conference Series. 132, 15. (1998)

9. P.Boissel, P.de Parseval, P.Marty, G.Lefebvre, J.Chem.Phys. 106, 4973 (1997)

10. L.Verstraete, A.Leger, L.d'Hendecourt, D.Defourneau, O.Dutuit, A\&A237, 436 (1990)

11. L.d'Hendecourt, M.Jourdain de Muizon, E. Dartois, K Demik, P. Ehrenfreund, A. Heras, in"The universe as seen by ISO", ESA SP-427, P. Cox and M.F. Kessler edit. (1999)

12. E.Pantin, P.O.Lagage, P.Artymowicz, A\&A, 327, 1123 (1997)

13. A.Abergel et al., A\&A, 315, L329 (1996); S.Bontemps et al., ASP Conference Series. 132, 141 (1998)

14. N.Grosso, T.Montmerle, E.D.Feigelson, P.André, S.Casanova, J.Gregorio-Hetem, Nature 387 (1997)

15. A.Sternberg, Min Yan, A.Dalgarno, in Molecules in Astrophysics: Probes and Processes, edited by E.F.van Dishoek (Kluwer1997) p. 141

16. S.Gougeon, Thèse, Université Paris 7, (1998)

1 Le Programme National sur la Physique et la Chimie du Milieu Interstellaire est une action CNRS inter-Départements (Sciences de l'Univers, Sciences Physiques et Mathématiques, Sciences Chimiques), pilotée par l'INSU, avec la participation contractuelle du CEA (Sciences de la Matière) et le soutien financier du CNES, créée en 1997 afin de poursuivre l'action du GDR "Physique et Chimie des Molécules et des Grains Interstellaires". Site Web: http://www.ias.fr/pcmi/ 